\title{
The Impact of Serum Lipid Levels on Circulating Soluble Adhesion Molecules in Childhood
}

\author{
EMMANUEL KAVAZARAKIS, MARIA MOUSTAKI, DIMITRIOS GOURGIOTIS, PETROS M. ZEIS, \\ APOSTOLOS BOSSIOS, ANTONIA MAVRI, AGELLIKI CHRONOPOULOU, AND \\ THEMISTOCLES KARPATHIOS \\ 2nd Department of Paediatrics, Athens University Medical School, "P and A Kyriakou" Children's \\ Hospital [E.K., M.M., D.G., P.M.Z., A.B., A.M., T.K.] and Department of Biochemistry, "P and A \\ Kyriakou” Children's Hospital [A.C.], Thibon and Levadias, Athens 115-27
}

\section{ABSTRACT}

\begin{abstract}
Cell adhesion molecules play a rather important role in the development of atherosclerosis mediating the attachment of monocytes to the endothelium. It has also been well established that hyperlipidemia is a risk factor for atherosclerosis from childhood. The aim of this study was to investigate whether the soluble adhesion molecules correlate with the circulating lipid levels in children. The study population consisted of 107 children (64 boys, 43 girls) aged 6-13 y. Parental history of cardiovascular disease, age, gender, and anthropometric parameters were recorded in all children. Blood samples were obtained from every child following a 12-hour fasting period. Serum triglycerides, total cholesterol, and its fractions as well as plasma levels of $\mathrm{P}$ and $\mathrm{E}$ selectins and adhesion molecules SVCAM-1 and SICAM-1 were determined. After controlling for age and body mass index, both SVCAM-1 and sP-selectin levels were inversely associated with HDL values $(\mathrm{r}=-0.33, p=0.005$ and $\mathrm{r}=-0.39, p=$ 0.001 , respectively). A significant positive correlation was found between sVCAM-1 and triglycerides $(\mathrm{r}=0.48, p<0.001)$. An increment of $10 \mathrm{mg} / \mathrm{dL}$ of HDL corresponds to about $50 \%$ reduction of the odds for endothelial dysfunction whereas an
\end{abstract}

increment of $10 \mathrm{mg} / \mathrm{dL}$ of triglyceride levels indicates a more than 3-fold excess risk, using either sP-selectin or sVCAM-1 levels as a surrogate for the determination of endothelial dysfunction. We suggest that HDL-C and triglycerides correlate in a biologically plausible way with soluble adhesion molecules, which therefore could be considered as useful indicators of the process of preclinical atherosclerosis even from childhood. (Pediatr Res 52: 454-458, 2002)
BMI, body mass index

\section{Abbreviations}
HDL-C, high density cholesterol fraction
LDL-C, low density cholesterol fraction
sICAM-1, soluble intercellular adhesion molecule
sVCAM-1, soluble vascular adhesion molecule
sP-selectin, soluble P selectin
sE-selectin, soluble E selectin
TC, total cholesterol
TG, triglycerides

Cardiovascular disease is among the major causes of morbidity and mortality in industrialized countries (1). Although cardiovascular disease affects almost exclusively adults, atherosclerosis, which is its underlying process, originates from childhood (2). Early coronary atherosclerosis as demonstrated by the formation of fatty streaks in the aorta has been found in children even at the age of 3 years (2). However, atherogenesis begins even earlier as the formation of the plaques does not represent the earliest stage of atherosclerotic process (3). Leukocyte-endothelial interaction seems to be a key factor in the initiation and evolution of atherosclerotic lesions (4). This interaction is mediated by cell adhesion molecules, namely

Received March 23, 2001; accepted April 10, 2002.

Correspondence and request reprints: Maria Moustaki, M.D., 2nd Department of Paediatrics, Athens University Medical School, "P and A Kyriakou" Children's Hospital, Thibon and Levadias, Goudi, Athens 115-27; e-mail march193@hol.gr

DOI: 10.1203/01.PDR.0000025349.12010.24 intercellular adhesion molecule-1 (ICAM-1), vascular adhesion molecule-1 (VCAM-1), P and E selectins $(5,6)$. Several studies have shown an increased expression of adhesion molecules at areas of vascular constriction or on the endothelial surface of symptomatic plaques $(4,7,8)$. It has also been suggested that the circulating levels of adhesion molecules in adults with cardiovascular disease or at risk for atherosclerosis reflect the up-regulation of these molecules on the endothelium $(9,10)$.

Although endothelial dysfunction has been shown in children with hypercholesterolemia $(11,12)$, a possible association of soluble adhesion molecules with serum lipid levels, a wellestablished risk factor for atherosclerosis even not clinically apparent, has not been adequately investigated in children. However, taking into consideration that adhesion molecules mediate the process of atherosclerosis at its very early stages, it is reasonable to assume that soluble adhesion molecules may 
be correlated to the circulating lipid levels in pediatric population. The aim of this study was to investigate whether and which of the adhesion molecules are related to the serum lipid profile in phenotypically healthy children.

\section{SUBJECTS AND METHODS}

The study participants were recruited from a primary school in the greater Athens area. A total of 107 children (64 boys and 43 girls) aged 6-13 y were studied. Eligible subjects were phenotypically healthy, had not either a chronic disease or a history of even a minor illness over the last 2-month period and were not on any medication. Clinical examination and data collection were performed by a pediatrician participating in the study. The following data were recorded: parental history of cardiovascular disease, age, gender, anthropometric parameters (weight, height, triceps skin-fold thickness), and Tanner stage of pubertal maturation. Parental history of cardiovascular disease was based on a healthy history questionnaire given to the parents of the participating children. A positive family history of cardiovascular disease was defined as myocardial infarction, bypass surgery, balloon angioplasty, angina, or hypertension at an age less than $55 \mathrm{y}$ in either a biologic parent or grandparent. Standing height was measured with a precision of $0.5 \mathrm{~cm}$ using a portable standard stadiometer, weight was measured in kilograms using a digital scale while children were on light clothes, without shoes. Triceps skin-fold was measured by a skin-fold calipers always on the left side of the body. All measurements were performed in duplicate and the average of the two readings was recorded. Body mass index $\left(\mathrm{kg} / \mathrm{m}^{2}\right)$ was calculated from the average of the corresponding measurements. The children were instructed to fast for $12 \mathrm{~h}$ before examination and two blood samples, appropriate for serum and plasma collection, were taken in the morning between 8:00 am and 10:00 am. The study was approved by the Health Committee of the Ministry of Education and an informed parental consent was obtained from all the participating subjects.

Serum and plasma was separated by centrifugation and then stored at $-70^{\circ} \mathrm{C}$ until analyzed. Serum total cholesterol, triglycerides, high-density cholesterol fraction (HDL-C), and soluble cell surface adhesion molecules (sICAM-1, sVCAM-1, sP-selectin, and sE-selectin) were determined in all the study participants. Triglycerides, total cholesterol and HDL-C were measured by an enzymatic colorimetric method using the BM Roche/Hitachi 717 analyzer (kits of Roche). Low-density cholesterol fraction (LDL-C) was calculated using the formula LDL-C $=$ Total cholesterol-HDL-C - Triglycerides/5 (13). Soluble adhesion molecules were measured in duplicate using commercially available kits from Biosource International Inc. and R\&D Systems, Inc. The interassay and the intra-assay coefficient of variation for soluble adhesion molecule determination were as follows: sICAM-1 8\%/6\%, sVCAM-1 7\%/5\%, sP-selectin $9 \% / 5.6 \%$, sE-selectin $8.5 \% / 5 \%$.

\section{STATISTICAL ANALYSIS}

Anthropometric variables, lipid values and soluble adhesion molecule levels were normally distributed. $t$ test, $\chi^{2}$, Pearson correlation, and multiple logistic regression models were used for data analysis as appropriate (14). A $p$ value $<0.05$ was considered statistically significant.

\section{RESULTS}

Demographic and anthropometric details of the study population are presented in Table 1. It is of importance to note that the 95th centile for BMI was 27.9, far less than 30, a value which has been suggested as a cutoff point for the identification of grade 2 overweight in adults, in children and adolescents (15). A BMI of 25 approximates the 90th percentile of the study population, indicating that even grade 1 overweight is encountered in about $10 \%$ of the participating subjects. A positive family history of cardiovascular disease was identified in 26 children.

Serum lipid profile and soluble adhesion molecule concentrations are shown in Table 2. The mean values of serum adhesion molecules, irrespective of the studied molecule, did not differ between children with total cholesterol below or above the 50th percentile. Similarly no difference was identified when the study population was split into two groups based on their LDL levels (group 1 with $\mathrm{LDL} \leq 2.71 \mathrm{mmol} / \mathrm{L}$ and group 2 with LDL $>2.71 \mathrm{mmol} / \mathrm{L}$ ). Plasma soluble VCAM-1 and P-selectin levels were significantly higher in children with HDL concentrations $\leq 1.45 \mathrm{mmol} / \mathrm{L}$ (median value for HDL) compared with those with HDL levels above the 50th centile ( $p$ $=0.025$ for sVCAM- 1 and $p<0.001$ for sP-selectin). The mean levels of soluble adhesion molecules were also elevated among children with triglyceride values higher than 0.65 $\mathrm{mmol} / \mathrm{L}$ (median value for triglycerides) but this difference reached a significant level only for $\operatorname{sVCAM}-1(p=0.036)$, whereas the significance for $\mathrm{sP}$ selectin was marginal $(p=$ 0.053). It was shown by other investigators that circulating adhesion molecules are age dependent and tend to decline with age over the age range of childhood and adolescence. However, the subjects of each pair of the above four pairs of groups did not differ significantly with respect to age distribution and parental history of cardiovascular risk. Therefore, the identified differences of the circulating adhesion molecule levels could not be attributed to confounding of age or family history.

Association of each one of the studied molecules with the levels of lipid parameters (one parameter at a time) was also assessed with Pearson correlation after controlling for age and BMI. Both sVCAM-1 and sP-selectin levels were inversely associated with HDL values $(\mathrm{r}=-0.33, p=0.005$ and $\mathrm{r}=$ $-0.39, p=0.001$, respectively). In contrast significant positive correlation was observed between sVCAM-1 concentrations and triglyceride values $(\mathrm{r}=0.48, p<0.001)$ whereas for sP-selectin the respective association was also positive $(\mathrm{r}=$ $0.23)$ but did not reach a significant level $(p=0.059)$. Circu-

Table 1. Anthropometric details of the 107 study participants

\begin{tabular}{lcccc}
\hline & Age (years) & Weight $(\mathrm{kg})$ & Height $(\mathrm{cm})$ & BMI $\left(\mathrm{kg} / \mathrm{m}^{2}\right)$ \\
\hline Mean & 9.2 & 37.4 & 175.8 & 20.5 \\
Median & 9 & 37.1 & 170.5 & 20.2 \\
$5^{\text {th }}$ centile & 7.3 & 20.1 & 115.3 & 15.1 \\
$9^{\text {th }}$ centile & 12.0 & 58.4 & 151.4 & 27.9 \\
SD & 1.7 & 11.3 & 10.5 & 4.1 \\
\hline
\end{tabular}


Table 2. Mean values and selected centiles of serum lipids and adhesion molecule levels in 107 phenotypicaly healthy children

\begin{tabular}{lcccccrrr}
\hline & $\begin{array}{c}\text { TC } \\
(\mathrm{mmol} / \mathrm{l})\end{array}$ & $\begin{array}{c}\text { TG } \\
(\mathrm{mmol} / \mathrm{l})\end{array}$ & $\begin{array}{c}\text { HDL-C } \\
(\mathrm{mmol} / \mathrm{l})\end{array}$ & $\begin{array}{c}\text { LDL-C } \\
(\mathrm{mmol} / \mathrm{l})\end{array}$ & $\begin{array}{c}\text { sICAM-1 } \\
(\mathrm{ng} / \mathrm{ml})\end{array}$ & $\begin{array}{c}\text { sVCAM-1 } \\
(\mathrm{ng} / \mathrm{ml})\end{array}$ & $\begin{array}{c}\text { SP-selectin } \\
(\mathrm{ng} / \mathrm{ml})\end{array}$ & $\begin{array}{c}\text { SE-selectin } \\
(\mathrm{ng} / \mathrm{ml})\end{array}$ \\
\hline Mean & 4.55 & 0.66 & 1.49 & 2.76 & 232.7 & 1008.5 & 200.1 \\
SD & 0.67 & 0.22 & 0.40 & 0.60 & 53.4 & 254.9 & 82.6 \\
Percentile & & & & & & & \\
$\quad 5^{\text {th }}$ & 3.61 & 0.28 & 0.93 & 1.86 & 161.5 & 591.6 & 87.1 \\
$5^{\text {th }}$ & 4.41 & 0.65 & 1.45 & 2.71 & 225.7 & 984.1 & 186.5 \\
$75^{\text {th }}$ & 5.10 & 0.79 & 1.72 & 3.20 & 262.5 & 1158.1 & 246.5 \\
$95^{\text {th }}$ & 5.83 & 1.19 & 2.33 & 3.96 & 333.9 & 1511.9 & 343.9 & 69.0 \\
\hline
\end{tabular}

lating sICAM-1 levels as well as sE-selectin were not related to any of the lipid profile variables.

Multiple logistic regression was used to evaluate the risk for endothelial dysfunction as this was reflected either by $\mathrm{sV}$ CAM-1 or by sP-selectin levels for a specified increase (10 $\mathrm{mg} / \mathrm{dL})$ of HDL $(0.26 \mathrm{mmol} / \mathrm{L})$ or triglyceride $(0.11 \mathrm{mmol} / \mathrm{L})$ levels after controlling for the mutually confounding effect of these variables as well as for the possible confounding influences of age ( 2 y increment), gender, BMI, and family history of cardiovascular disease. Two different logistic regression models were evaluated. In both models the dependent variable was the risk for endothelial dysfunction (low or high). In the first model sVCAM-1 levels were considered as a surrogate for the endothelial dysfunction (people at low risk those with sVCAM-1 levels below the corresponding 75 th percentile). In the second model the principle was similar, using, however, $\mathrm{sP}$-selectin levels as a surrogate for the endothelial dysfunction (people at low risk those with sP-selectin levels below the corresponding 75 th percentile). The 75 th centile was arbitrarily but not irrationally selected as the critical level for possible endothelial dysfunction taking into consideration that the population above this centile represents the upper quartile of the studied sample. The effect of triglycerides and HDL on sICAM-1 or sE-selectin was not evaluated through logistic analysis as correlation Pearson test and student's $t$ tests did not reveal any association of these variables with serum lipid profile.

Table 3 shows multiple logistic derived odds ratios for endothelial dysfunction in relation to HDL and triglyceride levels. An increment of $10 \mathrm{mg} / \mathrm{dL}$ of HDL corresponds to a substantial and significant reduction of the odds for endothelial dysfunction by $55 \%$ or $65 \%$ depending on the indicator that was used for the determination of endothelial dysfunction (sP-selectin and sVCAM-1, respectively). The protective effect of HDL levels is also depicted in Figures 1 and 2, which show the predicted probabilities for endothelial dysfunction, as this is represented by each surrogate, in relation to HDL levels. In contrast an increment of $10 \mathrm{mg} / \mathrm{dL}$ of triglyceride levels indicates a more than 3 -fold excess risk for having circulating

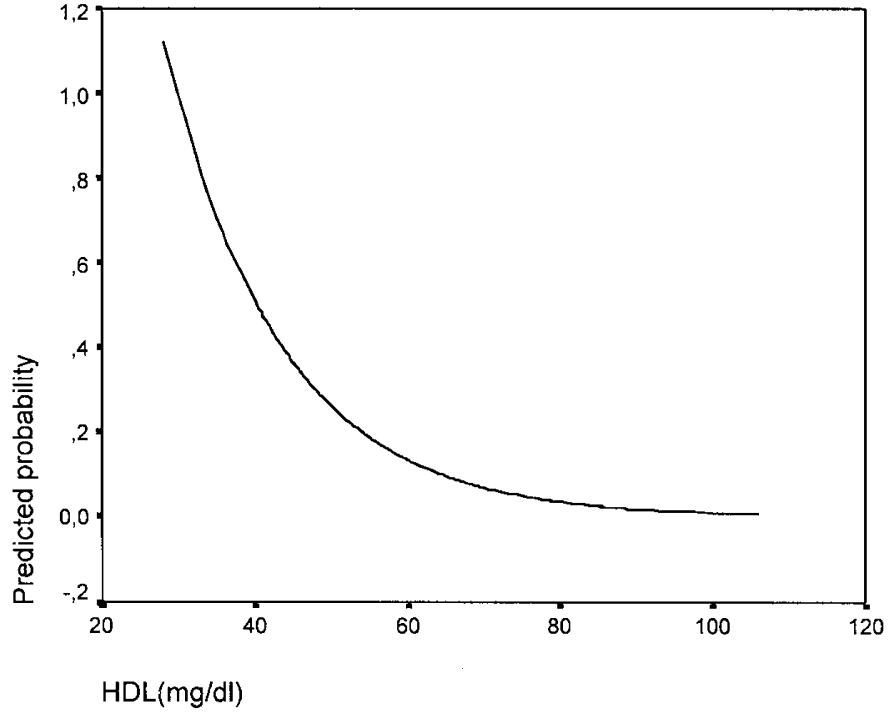

Figure 1. Predicted probability for endothelial dysfunction (surrogate: sPselectin levels $>75$ th centile) in relation to HDL values derived from the logistic regression analysis

sVCAM-1 levels above the 75th percentile (Fig. 3). No significant association was found between triglycerides and endothelial dysfunction when sP-selectin was used as surrogate.

\section{DISCUSSION}

It has been well established that hyperlipidemia plays a key role in the development of cardiovascular disease in adulthood $(16-18)$ and that lowering lipid levels leads to significant reduction of cardiovascular heart events and mortality $(19,20)$. No longitudinal studies have been conducted to address directly the question whether cholesterol levels in childhood correlate with cardiovascular disease in adulthood. However, considerable indirect evidence indicates that cholesterol levels in childhood are associated with the atherosclerotic process (21). The identification therefore of a relationship of easily determined indicators of atherosclerosis with serum lipid profile in childhood would allow clinicians to follow not only the

Table 3. Multiple logistic derived odds ratios and 95\% confidence intervals for endothelial dysfunction by serum levels of HDL and triglycerides after controlling for gender, age, BMI and family history of cardiovascular risk

\begin{tabular}{clcc}
\hline Surrogate for endothelial dysfunction & \multicolumn{1}{c}{ Variable } & ORs & 95\% CI \\
\hline 1st regression model & HDL (increment $10 \mathrm{mg} / \mathrm{dl}=0.26 \mathrm{mmol} / \mathrm{l})$ & 0.45 & $0.24-0.86$ \\
$75^{\text {th }}$ centile of sP-selectin & TG (increment $10 \mathrm{mg} / \mathrm{dl}=0.11 \mathrm{mmol} / \mathrm{l})$ & 1.28 & $0.92-1.77$ \\
2nd regression model & HDL (increment $10 \mathrm{mg} / \mathrm{dl}=0.26 \mathrm{mmol} / \mathrm{l})$ & 0.27 & $0.09-0.77$ \\
$75^{\text {th }}$ centile of sVCAM-1 & TG (increment $10 \mathrm{mg} / \mathrm{dl}=0.11 \mathrm{mmol} / \mathrm{l})$ & 3.22 & $1.40-7.43$ \\
\hline
\end{tabular}




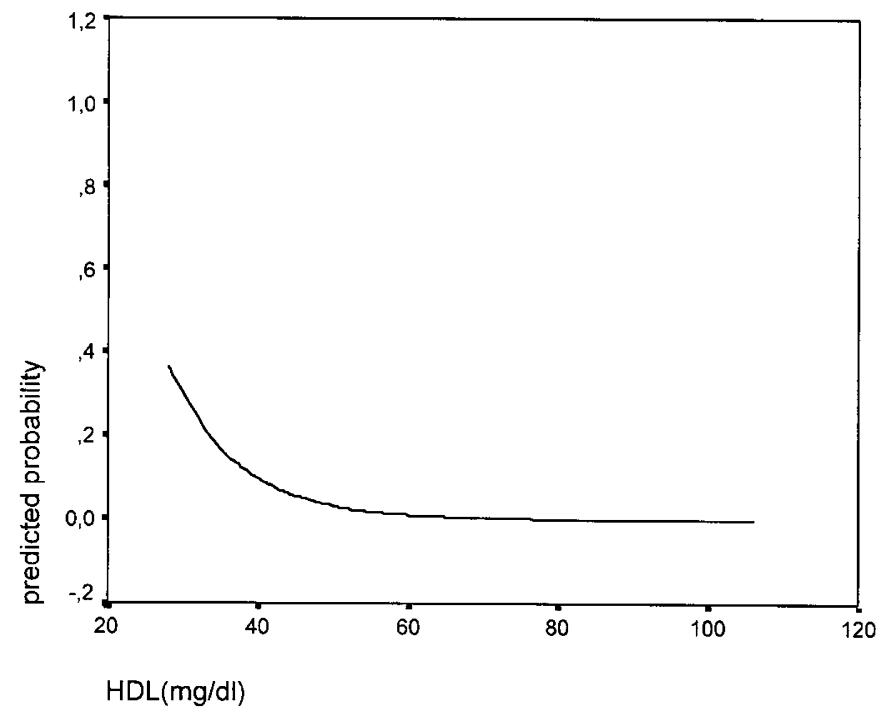

Figure 2. Predicted probability for endothelial dysfunction (surrogate: sVCAM-1 levels $>75$ th centile) in relation to HDL values derived from the logistic regression analysis.

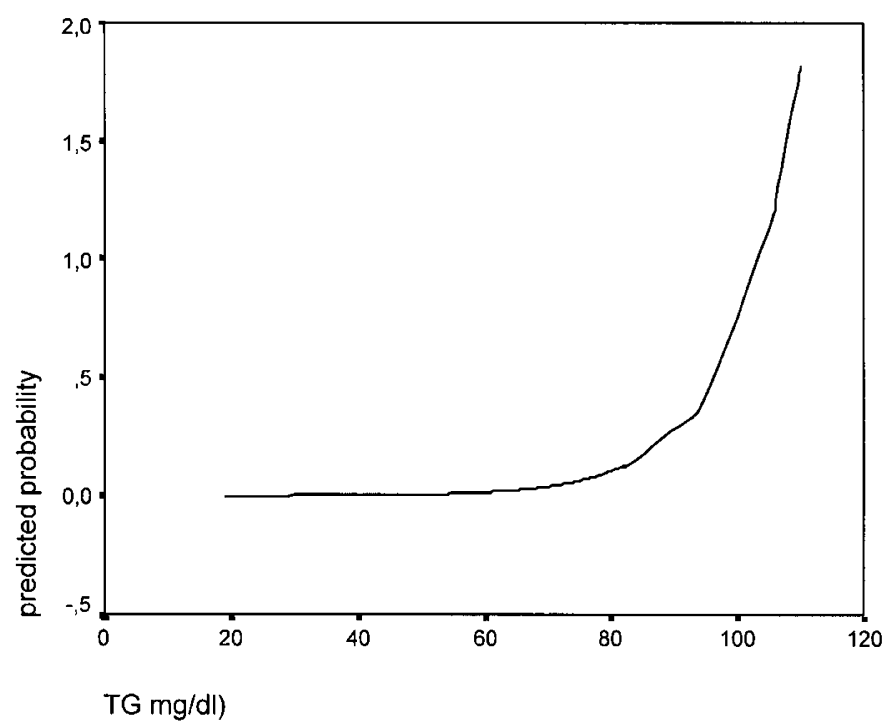

Figure 3. Predicted probability for endothelial dysfunction (surrogate: sVCAM-1 levels $>75$ th centile) in relation to triglyceride values, derived from the logistic regression analysis.

serum lipid levels in children but also the evolution of their atherosclerotic process, modifying accordingly the therapeutic interventions based not only on the lipid values but also on indicators of atherosclerosis.

Our study despite the rather limited number of participants allowed the identification of a significant relationship between certain lipid fractions with SVCAM-1 and sP-selectin circulating levels. The fact that the study population consisted of phenotypically healthy children with no previous history of hospitalization or of any other type of disease that may have influenced the adhesion molecule levels and controlling for confounding performed through the regression analysis ensure the validity of our results. Our results are biologically plausible as triglycerides, which are a known risk factor for atherosclerosis, were found to increase the odds for endothelial dysfunc- tion. Although one could argue that our population had triglyceride levels within the normal values, the association we found is still reasonable as adhesion molecules play an important role even in early atherogenesis preceding the appearance of foam cells in the arterial intima (22), a process unavoidable even in healthy people. Furthermore, HDL cholesterol fraction, which is considered a protective factor against coronary heart disease $(1,4,18)$, reduced the probability of endothelial dysfunction in our population. This finding is also in accordance with experimental evidence showing that HDL inhibits the cytokineinduced expression of endothelial cell adhesion molecules (23).

The question that is reasonably raised is whether and to what extent soluble adhesion molecule levels above the corresponding 75 th centile reflect endothelial dysfunction. It was obviously beyond the objective and the ability of our study design to address this issue. However, the identification of a biologically plausible association of certain cell adhesion molecules with the lipid profile in childhood indicates the need for further studies in children population which will determine the level of soluble adhesion molecules that corresponds to endothelial dysfunction as this can be detected directly by noninvasive high resolution ultrasound techniques. To the best of our knowledge there are no relevant studies in children, exploring this association. The findings of recent studies in adults (24, 25 ) are in support of our suggestion as they provided evidence of a significant concordance between the two measures of endothelial dysfunction (ultrasonographic and biochemical, respectively).

Our data revealed that only SVCAM-1 and sP-selectin levels were associated with lipid concentrations. These findings are in part and not entirely consistent with recently provided evidence by Ohta et al. (26) in a childhood Japanese population. In that study an association of sVCAM-1 and sICAM-1 and serum lipid profile was identified whereas sE-selectin values were not determined and sP-selectin levels did not correlate with any lipid fraction. Furthermore, in our study, gender was not a significant determinant of the associations we found, whereas in the Japanese population different lipid fractions accounted for the variability of soluble adhesion molecule levels in the male and female population, respectively. However, as the regulating factors of cell adhesion molecules have not been entirely elucidated it would not be justified to attempt comparisons between populations of different descent (Caucasians and non-Caucasians) with all the genotypical and environmental inferences of that difference.

In conclusion, we suggest that soluble adhesion molecule levels correlate in a biologically plausible way with the circulating lipid values in children. Additional studies are clearly needed to establish normal values for adhesion molecules in childhood. This determination would allow the identification of children who would be mostly benefited by the implementation of preventive and therapeutic programs aiming at the lipid reduction or the modification of other established risk factors for atherosclerosis in childhood in an attempt to decelerate the evolution of atherosclerotic process. 


\section{REFERENCES}

1. Genest J Jr, Cohn JS 1995 Clustering of cardiovascular risk factors: targeting high-risk individuals. Am J Cardiol 76(suppl)8A-20A

2. Stary HC 1989 Evolution and progression of atherosclerotic lesions in coronary arteries in children and young adults. Atherosclerosis 99(suppl I):19-32

3. Ross R 1999 Atherosclerosis-An inflammatory disease. N Engl J Med 340:115-126

4. Frishman WH 1998 Biologic markers as predictors of cardiovascular disease. Am J Med 104:18S-27S

5. Carlos TM, Harlan JM 1994 Leucocyte-endothelial adhesion molecules. Blood $84: 2068-2101$

6. Ross R 1993 The pathogenesis of atherosclerosis: a perspective for the 1990s. Nature 362:801-809

7. Poston RN, Haskard DO, Coucher JR, Gall NP, Johnson-Tidey RR 1992 Expression of intercellular adhesion molecule-1 in atherosclerotic plaques. Am J Pathol 140:665673

8. Richardson M, Hadcock SJ, DeReske M, Cybulsky MI 1994 Increased expression in vivo VCAM-1 and E-selectin by the aortic endothelium of normolipidemic and hyperlipidemic diabetic rabbits. Arterioscler Thromb 14:760-769

9. Hackman A, Yasunori A, Insull W Jr, Pownall H, Smith L, Dunn K, Gotto AM Jr, Ballantyne CM 1996 Circulation levels of soluble cell adhesion molecules in patients with dyslipidemia. Circulation 93:1334-1338

10. Mulvihill N, Foley JB, Ghaisas N, Murphy R, Crean P, Walsh M 1999 Early temporal expression of soluble cellular adhesion molecules in patients with unstable angina and subendocardial myocardial infarction. Am J Cardiol 83:1265-1267

11. Celermajer DS, Sorensen KE, Gooch VM, Spiegelhalter DJ, Miller OW, Sullivan ID, Lloyd JK, Deanfield JE 1992 Non-invasive detection of endothelial dysfunction in children and adults at risk of atherosclerosis. Lancet 340:1111-1115

12. Mietus-Snyder M, Malloy MJ 1998 Endothelial dysfunction occurs in children with two genetic hyperlipidemias: Improvement with antioxidant vitamin therapy. J Pediatr 133:35-40

13. Friedewald WT, Lewy RI, Fredrickson DS 1972 Estimation of the concentration of low-density lipoprotein cholesterol plasma, without use of the preparative ultracentrifuge. Clin Chem 18:499-502
14. MacMahon B, Trichopoulos D 1996 Epidemiology Principles and Methods. Little Brown and Company, Boston, pp 290-291

15. Dietz WH 1998 Use of the body mass index (BMI) as a measure of overweight in children and adolescents. J Pediatr 132:191-193

16. Krauss RM 1987 Relationship of intermediate and low-density lipoprotein subspecies to risk of coronary artery disease. Am Heart J 113:578-582

17. Kannel WB 1985 Lipids, diabetes, and coronary heart disease: insights from the Framingham Study. Am Heart J 110:1100-1106

18. O' Keefe JH Jr, Lavie CJ Jr, McCallister BD 1995 Insights into the pathogenesis and prevention of coronary artery disease. Mayo Clin Proc 70:69-79

19. Libby P, Aikawa M, Kinlay S, Selwyn A, Ganz P 1999 Lipid lowering improves endothelial functions. Int J Cardiol 74:S3-S10

20. Robinson JG, Leon AS 1994 The prevention of cardiovascular disease. Med Clin North Am 78:69-99

21. American Academy of Paediatrics Committee on Nutrition 1998 Cholesterol in childhood. Paediatrics 101:141-147

22. Li H, Cybulsky MI, Gimbrone MA Jr, Libby P 1993 An atherogenic diet rapidly induces VCAM-1, a cytokine-regulatable mononuclear leukocyte adhesion molecule, in rabbit aortic endothelium. Arterioscler Thromb 13:197-204

23. Cockerill GW, Rye KA, Gamble JR, Vadas MA, Barter PJ 1995 High- density cytokines inhibit cytokine induced expression of endothelial cell adhesion molecules. Arterioscler Thromb Vasc Biol 15:1987-1994

24. Lupatelli G, Lombardini R, Schillaci G, Giuffetti G, Marchesi S, Siepi D, Mannarino E 2000 Flow-mediated vasoactivity and circulating adhesion molecules in hypertriglyceridemia: Association with small, dense LDL cholesterol particles. Am Heart J 140:521-526

25. Rohde LE, Lee RT, Rivero J, Jamacochian M, Arroyo LH, Briggs W, Rifai N, Libby P, Creager MA, Ridker PM 1998 Circulating cell adhesion molecules are correlated with ultrasound- based assessment of carotid atherosclerosis. Arterioscler Thromb Vasc Biol 18:1765-1770

26. Ohta T, Saku K, Takata K, Adachi N 1999 Soluble vascular cell-adhesion molecule-1 and soluble intercellular adhesion molecule-1 correlate with lipid and apolipoprotein risk factors for coronary artery disease in children. Eur J Pediatr 158:592-598 